\title{
Exogenous melatonin inhibits neutrophil migration through suppression of ERK activation
}

\author{
Da-Long Ren, Ai-Ai Sun, Ya-Juan Li, Min Chen, Shu-Chao Ge and Bing Hu
}

Chinese Academy of Sciences Key Laboratory of Brain Function and Disease, School of Life Sciences, University of Science and Technology of China, No. 96 Jinzhai Road, Hefei, Anhui Province 230026, People's Republic of China

Correspondence should be addressed to B Hu

Email

bhu@ustc.edu.cn

\begin{abstract}
Neutrophil migration to inflammatory sites is the fundamental process of innate immunity among organisms against pathogen invasion. As a major sleep adjusting hormone,

melatonin has also been proved to be involved in various inflammatory events. This study aimed to evaluate the impact of exogenous melatonin on neutrophil migration to the injury site in live zebrafish and further investigate whether ERK signaling is involved in this process. Using the tail fin transection model, the fluorescently labeled neutrophil was in vivo visualized in transgenic $\mathrm{Tg}(/ y z$ :EGFP), $\mathrm{Tg}$ (lyz:DsRed) zebrafish. We found that exogenous melatonin administration dramatically inhibited the injury-induced neutrophil migration in a dose-dependent and time-dependent manner. The inhibited effect of melatonin on neutrophil migration could be attenuated by melatonin receptor 1,2 , and 3 antagonists. The ERK phosphorylation level was significantly decreased post injury when treated with melatonin. The blocking of ERK activation with inhibitor PD0325901 suppressed the number of migrated neutrophils in response to injury. However, the activation of ERK with the epidermal growth factor could impair the inhibited effect of melatonin on neutrophil migration. We also detected that PD0325901 significantly suppressed the in vivo neutrophils transmigrating over the vessel endothelial cell using the transgenic $\operatorname{Tg}(f l k: E G F P) ;(l y z: D s R e d)$ line labeled as both vessel and neutrophil. Taking all of these data together, the results indicated that exogenous melatonin had an anti-migratory effect on neutrophils by blocking the ERK phosphorylation signal, and it led to the subsequent adhesion molecule expression. Thus, the crossing of the vessel endothelial cells of neutrophils became difficult.
\end{abstract}

Key Words
- melatonin
- neutrophil
- injury
- MAPK/ERK
- migration
- zebrafish

Journal of Endocrinology (2015) 227, 49-60

\section{Introduction}

Melatonin, also called the 'darkness hormone,' is synthesized from tryptophan by aralkylamine $N$-acetyltransferase (aanat1 and aanat2), mostly in the pineal and retina cells (Falcon et al. 2001, Appelbaum et al. 2006, LimaCabello et al. 2014). Aanat1 is mainly expressed in the retina, and aanat 2 is distributed in both the pineal and the retina (Appelbaum et al. 2006). As the major output of the pineal gland in vertebrates, melatonin plays a vital homeostatic role in the rhythm-dependent basic functions of zebrafish physiology, including sleep (Zhdanova 2011, Elbaz et al. 2013), diet (Lima-Cabello et al. 2014), development (Kazimi \& Cahill 1999, de Borsetti et al. 2011), and reproduction (Reiter et al. 1976, Barrett \& Bolborea 2012) and also in neural functions such as

Published by Bioscientifica Ltd 
learning and memory (Baydas et al. 2005, Moriya et al. 2007). Almost all of the functional actions of the melatonin system are conducted through the identified receptor 1, 2, or 3 (Fusani \& Gahr 2014, Kleber et al. 2014, Lee et al. 2014). In zebrafish, melatonin and its receptors become functional at $\sim 20 \mathrm{~h}$ post-fertilization (Danilova et al. 2004). Moreover, the identified melatonin receptors $1 \mathrm{a}, 1 \mathrm{~b}$, and $1 \mathrm{c}$ of zebrafish are highly homologous to those found in mammals and amphibians (Reppert et al. 1995, Masana \& Dubocovich 2001), namely 1a and 1b (corresponding to 1 and 2 respectively) and 1c (corresponding to 3). Except for the abovementioned characters, a growing number of investigations have proved that melatonin also acts as an important modulator in many immune-related physiology processes.

In the nervous and endocrine systems, melatonin has been suggested to interact with the immune system to modulate its function (Blalock 1994). Leukocytes contain specific melatonin receptors, including MT1, MT2, and nuclear RZR (retinoid $\mathrm{Z}$ receptor), which give a molecular basis for the perception of leukocytes on melatonin (Radogna et al. 2010). Melatonin has been demonstrated to affect the rhythm of leukocytes, cytokines production (Delgobbo et al. 1989, Scheff et al. 2010), and NK cell activity (Matsumoto et al. 2001) in mammalian bone marrow cells. In other studies, pineal removal further elucidated the effect of melatonin on the immune system. Pineal excision leads to a partial immune function deficiency, such as decreased IL-2 production (Delgobbo et al. 1989), impaired colony forming units of granulocytes and macrophages from rat bone marrow cell cultures (Haldar et al. 1992), and changed allergic lung inflammation (Martins et al. 2001). All previous studies confirmed the considerable role of melatonin in immune system stabilization.

Recently, melatonin has also been proved to modulate leukocyte behavior in physiological chemoattractants, but the studies were short of consistently functional results (Lotufo et al. 2001, Pena et al. 2007). The inconsistent role of melatonin in previous studies indicates that the endogenous and exogenous melatonin may execute discrepant function in immune stabilization (delGobbo et al. 1989, Lotufo et al. 2001, Pena et al. 2007). Our latest study demonstrated that the pineal melatonin plays a positively regulating role in neutrophil migration (Ren et al. 2015), and this finding motivated us to further explore the potential effect of exogenous melatonin on neutrophil function. Thus, this study aimed to investigate, in vivo, the effect of exogenous melatonin on neutrophil migration to the injury site and the underlying regulating mechanism.

\section{Material and methods}

\section{Zebrafish lines and maintenance}

Zebrafish embryos were harvested from naturally matched wide-type $(\mathrm{AB})$, transgenic $\operatorname{Tg}($ lyz:EGFP), and $\operatorname{Tg}$ (lyz:DsRed) lines maintained in $28.5^{\circ} \mathrm{C}$. Under the control of the lysozyme $\mathrm{C}$ promoter, both $\mathrm{Tg}(l y z$ :EGFP) and $\operatorname{Tg}($ lyz:DsRed) lines labeled as neutrophil through expressing the enhanced green fluorescent protein (EGFP) and DsRed, respectively. $\operatorname{Tg}(f l k: \mathrm{EGFP})$ line labeled as the vessel endothelial cells with fluorescent protein EGFP, which was under the control of the flk promoter. To obtain the embryos labeled as both neutrophil and vessel, we hybridized the transgenic Tg(lyz:DsRed) (Liu \& Wen 2002) and $\mathrm{Tg}$ (flk:EGFP) (Choi et al. 2007) lines. Thus, the next generation of embryos expressed both fluorescent protein EGFP (labeling vessel) and DsRed (labeling neutrophil) with the lysozyme $C$ and flk promoter, respectively. All animal manipulations were conducted in strict accordance with the guidelines and regulations set by the University of Science and Technology of China (USTC) Animal Resources Center and the University Animal Care and Use Committee. The protocol was approved by the Committee on the Ethics of Animal Experiments of the USTC (Permit Number: USTCACUC1103013). Zebrafish surgery was performed under the solution of tricaine methane-sulfonate (MS-222, Sigma) for anesthesia, and all efforts were made to minimize suffering.

\section{Pharmacological treatment}

To evaluate the effect of melatonin on neutrophil migration, zebrafish were pretreated $1 \mathrm{~h}$ in the presence or absence of melatonin (Sigma, M5250) at different final concentrations via ambient water, and treatment during the injury period was maintained. MT1/MT2 (melatonin receptor $1 / 2)$ antagonist luzindole $(30 \mu \mathrm{M})$ (Sigma, L2407) and MT3 antagonist prazosin $(5 \mu \mathrm{g} / \mathrm{ml})$ (Sigma, P7791) were used to detect the role of the melatonin receptor in neutrophil migration. Either PD0325901 $(20 \mu \mathrm{M})$, an MEK inhibitor, (Sigma, PZ0162) or epidermal growth factor (EGF) $(1 \mu \mathrm{g} / \mathrm{ml})$ (Sigma, E9644) was applied $1 \mathrm{~h}$ before injury to downregulate or upregulate ERK activity respectively.

\section{Tail fin injury and live imaging}

After being anesthetized with tricaine methane-sulfonate (Sigma), zebrafish tail fin was cut at the end of the spinal

Published by Bioscientifica Ltd. 
cord using a sterile scalpel blade on a plastic petri dish. The embryo was live imaged $3 \mathrm{~h}$ later by a fluorescence microscope (Olympus BX60) using a green fluorescent channel and a $10 \times$ lens. To determine the neutrophil migration speed and vessel-crossing evaluation, the larvae embedded with $1 \%$ agarose underwent time-lapse imaging using a confocal microscope (Olympus FV1000) from 30 to 60 min after injury.

\section{Immunocytochemistry}

A blood sample collected from adult transgenic $\operatorname{Tg}(l y z$ : EGFP) zebrafish heart was treated with melatonin (1.5 mM) and PD0325901 $(20 \mu \mathrm{M})$ to evaluate the p-ERK expression of neutrophils. Fresh blood was centrifuged at $800 \boldsymbol{g}$ for $10 \mathrm{~min}$, and the precipitate was used for immunocytochemistry analysis. The primary monoclonal antibody of p-ERK sourced from rabbit (Cell Signaling Technology (CST), 4379S, Boston, MA, USA) was diluted 1:300 for $2 \mathrm{~h}$ at room temperature, and the secondary antibody (goat anti-rabbit) (Invitrogen, A11036) was used at 1:300 for $1 \mathrm{~h}$. 4,6-diamino-2-phenyl indole (DAPI) diluted at 1:10 000 was used to stain the nucleus for $10 \mathrm{~min}$.

\section{Western blot}

The embryo was treated with melatonin $(1.5 \mathrm{mM})$, PD0325901 $(20 \mu \mathrm{M})$, and EGF $(1 \mu \mathrm{g} / \mathrm{ml})$ to analyze the p-ERK expression using western blot. The embryo was homogenized and centrifuged for protein collection $1 \mathrm{~h}$ after injury. The p-ERK (CST, 4379S) and ERK (CST, 4695S) primary antibodies sourced from rabbit were used at 1:1000 overnight at $4{ }^{\circ} \mathrm{C}$. $\beta$-actin (CST, 4967S) and p-Akt (CST, 4060) monoclonal antibodies sourced from rabbit was also diluted at 1:1000. The HRP-conjugated goat anti-rabbit secondary antibody (Sangon, D1110420100, Shanghai, China) was diluted at 1:10 000 for $2 \mathrm{~h}$ at room temperature.

\section{RT-PCR}

For the melatonin receptor evaluation, the melatonin receptor was analyzed by RT-PCR with a specific primer. Melatonin receptor 1a1: F-GGTGGTGACTTTACTCTCCAGCGT, R-ACCACCACCATATGGTATAAAGTGA; 1aa: F-GCGAGCAAGCACTTCAGCGGC, R-AGGAACAACCCGCTCTGGAGAAAT; 1ba: F-GGCGTGGGTCGTCATGGTACT, R-TCAAGAAATGGCGCAACTCACTAG; 1bb: F-ATTTGGGGCACGTCGGTCGC， R-CCACAGTGTAGGAGCTGCTGGCAGT; 1c: F-ACAGTCTTCGCTACGACCGACT,
R-GCTTCAGTGTTATACCTGGAACTGTC. Adhesion molecular analysis, E-selectin: F-CCTCACTGTGAAGCTGTGGT, R-TCATGGCTGCACATCACTGT; P-selectin: F-TTCATGGGTCCACGCTTTGT, R-CAGTTGCCTTCTCTCTGGCA; $\beta$-actin: F-CATTGGCAATGAGCGTTTC, R-TACTCCTGCTTGCTGATCCAC. After removing the erythrocyte of the fresh blood collected from the adult zebrafish heart, a leukocyte sample was used for RT-PCR to detect the melatonin receptor expression. Treated with PD0325901 and melatonin, 50 embryos were collected for the adhesion molecular analysis.

\section{In vitro migration of neutrophil using transwell assay}

The peripheral blood of mouse was collected into an anticoagulant tube. Using the isolation kit (Sangon, E501091), blood was divided into four liquid layers with $500 \mathrm{~g}$ for $20 \mathrm{~min}$. Collected cells from the third and fourth liquid layer were treated with red blood cell lysis buffer with $500 \mathrm{~g}$ for $30 \mathrm{~min}$. With the melatonin and lipopolysaccharide (Sigma) treatment, each hole was added with $100 \mu \mathrm{l}$ diluted cell at the number of $2 \times 10^{5} / \mathrm{ml}$. The migrated neutrophil was counted using crystal violet staining.

\section{Apoptosis assay using TUNEL}

Larvae were fixed with $4 \%$ paraformaldehyde for $2 \mathrm{~h}$ at room temperature. Using the TUNEL kit (Vazyme, Nanjing, China), the fixed embryos were stained overnight at $4{ }^{\circ} \mathrm{C}$ according to the manufacturer's instructions. After removing the staining solution and washing the embryos with PBS, the apoptosis cells were observed using a fluorescent microscope.

\section{Statistical analysis}

For neutrophils counting, we determined the area within $250 \mu \mathrm{m}$ from the wound ending as the target, because very few neutrophils were located in this region before injury. In vivo, the random three-dimensional movement of neutrophils in tissues between 30 and 60 min post injury was monitored using a confocal microscope as the former performance (Li et al. 2012). The area and major axis of the activated neutrophils at $1 \mathrm{~h}$ were measured using a previously reported method (Yoo et al. 2012). The analysis of neutrophil speed, area, and roundness was completed by Imaris (Bitplane, Zurich, Switzerland) and ImageJ (National Institutes of Health, Bethesda, MD, USA) Software. The dynamic movement of neutrophils was tracked

Published by Bioscientifica Ltd 
by Imaris Software using the surpass function. The speed was directly obtained from the Imaris Software, and the area was measured by ImageJ. The roundness of neutrophils at $1 \mathrm{~h}$ post wounding was calculated as $4 \times$ area $/ \pi \times$ major_axis ${ }^{2}$. The data were analyzed with unpaired, twotailed $t$-test and one-way ANOVA using GraphPad Prism version 5.0 (Prism, New York, NY, USA). The results were shown as mean \pm s.e.m. The level of significance was set to $P<0.05$. * ${ }^{* *}$ and ${ }^{* * *}$ represent $P<0.05, P<0.01$, and $P<0.001$, respectively.

\section{Results}

In obtaining a model for investigating neutrophil migration, the caudal fin of transgenic zebrafish $\operatorname{Tg}(l y z$ : EGFP) labeled as neutrophil was transected at the end of the spinal cord (Fig. 1A) according to our previous study (Ren et al. 2015). We in vivo monitored the aggregation and dissipation of neutrophils to the injury area (Fig. 1B). The neutrophils gradually arrived at the tail fin terminal, peaked at $\sim 6 \mathrm{~h}$, and subsequently dispersed out of sight (Fig. 1C). Using this model, we tested the role of melatonin in neutrophil migration at $3 \mathrm{~h}$ post injury instead of the peak time to avoid counting error, because too many neutrophils had accumulated in the tail fin terminal at $6 \mathrm{~h}$ post injury. The results showed that exogenous melatonin of different concentrations could significantly suppress neutrophil-directed migration conducted in a dosedependent manner (Fig. 1D and E). In vitro experiment also demonstrated the similar inhibitory effect of melatonin on neutrophil migration (Supplementary Figure 1A and $\mathrm{B}$, see section on supplementary data given at the end of this article). To exclude the factor such as loss of neutrophil-like apoptosis induced by melatonin, we did the apoptosis experiment in vivo. The results indicated that melatonin did not induce neutrophil apoptosis (Supplementary Figure 1C). Moreover, we observed the dynamic influence of melatonin on neutrophil migration. Melatonin also inhibited neutrophil migration in a time-dependent manner (Fig. 1F and G). The results

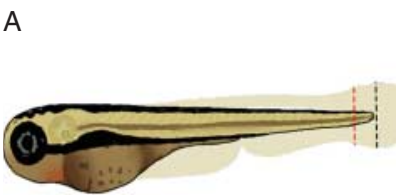

D

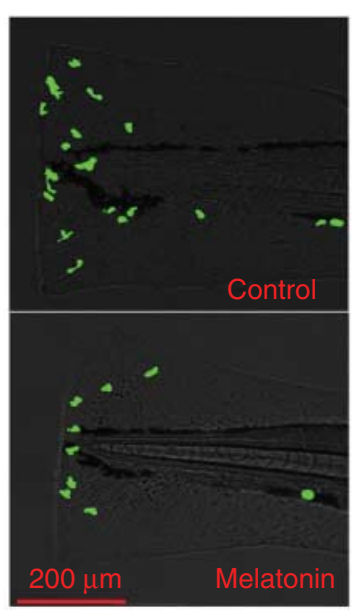

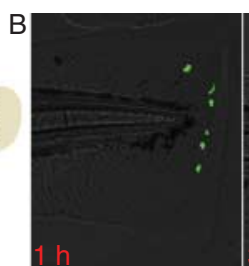
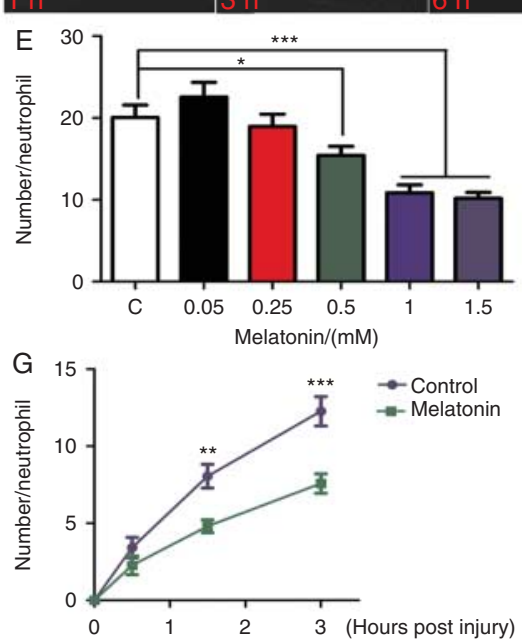
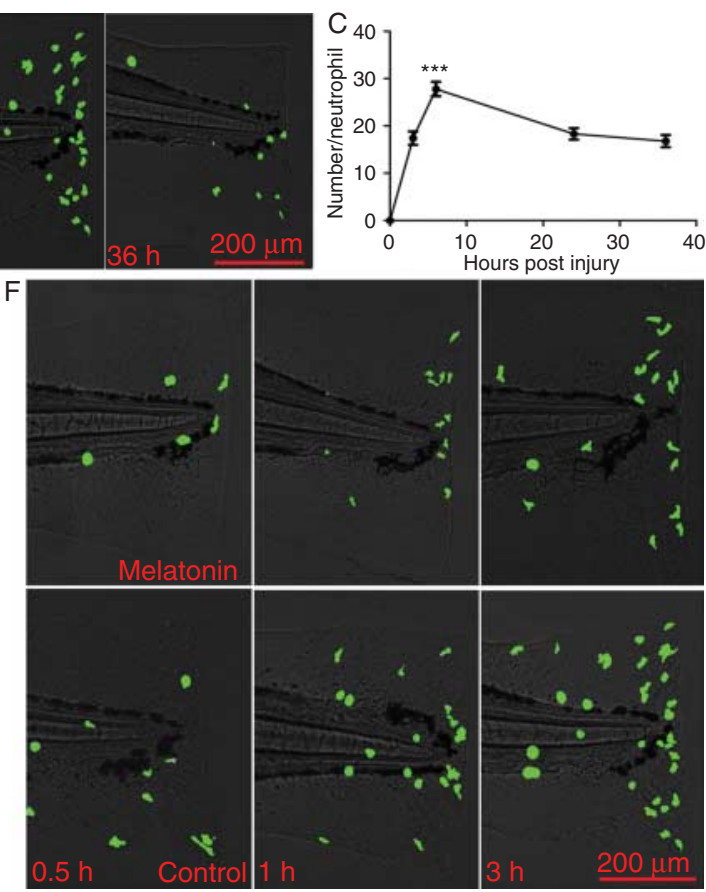

\section{Figure 1}

Melatonin inhibited injury-induced neutrophil migration. (A) Transgenic zebrafish Tg(lyz:EGFP) tail fin was transected at the end of the spinal cord by a sterile blade (indicated by black line). (B and C) In vivo imaging revealed the dynamic motion of neutrophils toward the injured tail fin. Recruitment started at $\sim 0.5 \mathrm{~h}$, peaked at $\sim 6 \mathrm{~h}$, and then gradually dismissed at $24 \mathrm{~h}$ post wounding. ( $D$ and $E$ ) Neutrophil recruitment was significantly suppressed by melatonin of different concentrations; $1.5 \mathrm{mM}$ melatonin caused the most obvious inhibition effect. ( $F$ and $G$ ) Melatonin acted on neutrophil migration in a time-dependent manner. Neutrophils located at $250 \mu \mathrm{m}$ from the wounded ending (between black and red line shown in (A)) were counted and regarded as the valid migration number, because few neutrophils located the area before injury $(* P<0.05$,

$* * P<0.01, * * * P<0.001, t$-test and ANOVA analysis). 
demonstrated that exogenous melatonin inhibits neutrophil recruitment to the injury site in a concentration- and time-dependent manner.

We also aimed to determine whether melatonin also disturbs the neutrophil migration activity in the tail fin tissues. We monitored the dynamic movement of neutrophils in the tail fin tissue after injury (Supplementary Figure 2, see section on supplementary data given at the end of this article). The average migration speed of neutrophils undergoing melatonin treatment had no significant difference with that of the control group (Fig. 2A and B). Subsequently, we focused on the effect of melatonin on the dynamic shape motility of neutrophils. The results showed that melatonin does not induce the notable area and roundness $\left(4 \times\right.$ area $/ \pi \times$ major_axis $\left.{ }^{2}\right)$ change in neutrophils (Fig. 2C, D and E). We then examined whether melatonin affects the phosphorylation of p-Akt, a cytoskeleton-related protein that could regulate neutrophil polarity (Tell et al. 2012, Germena \& Hirsch 2013). No detectable difference was observed at the western blot protein level of Akt phosphorylation when treated with exogenous melatonin (Fig. 2F and G). This finding could hint that melatonin was not involved in regulating neutrophil cytoskeleton remodeling. Taken together, melatonin most likely only impaired the directional neutrophil migration but did not affect the cell motility in the tail fin tissues.

In this section, we evaluate the potential of different receptors in neutrophil migration. We first identified the expression of multiple receptor subtypes in different tissues. The results indicated that melatonin receptors 1a1, 1aa, 1ba, 1bb, and 1c were all expressed in the whole zebrafish embryo, especially in leukocytes (Fig. 3A and B). Then, we used different melatonin receptor antagonists,
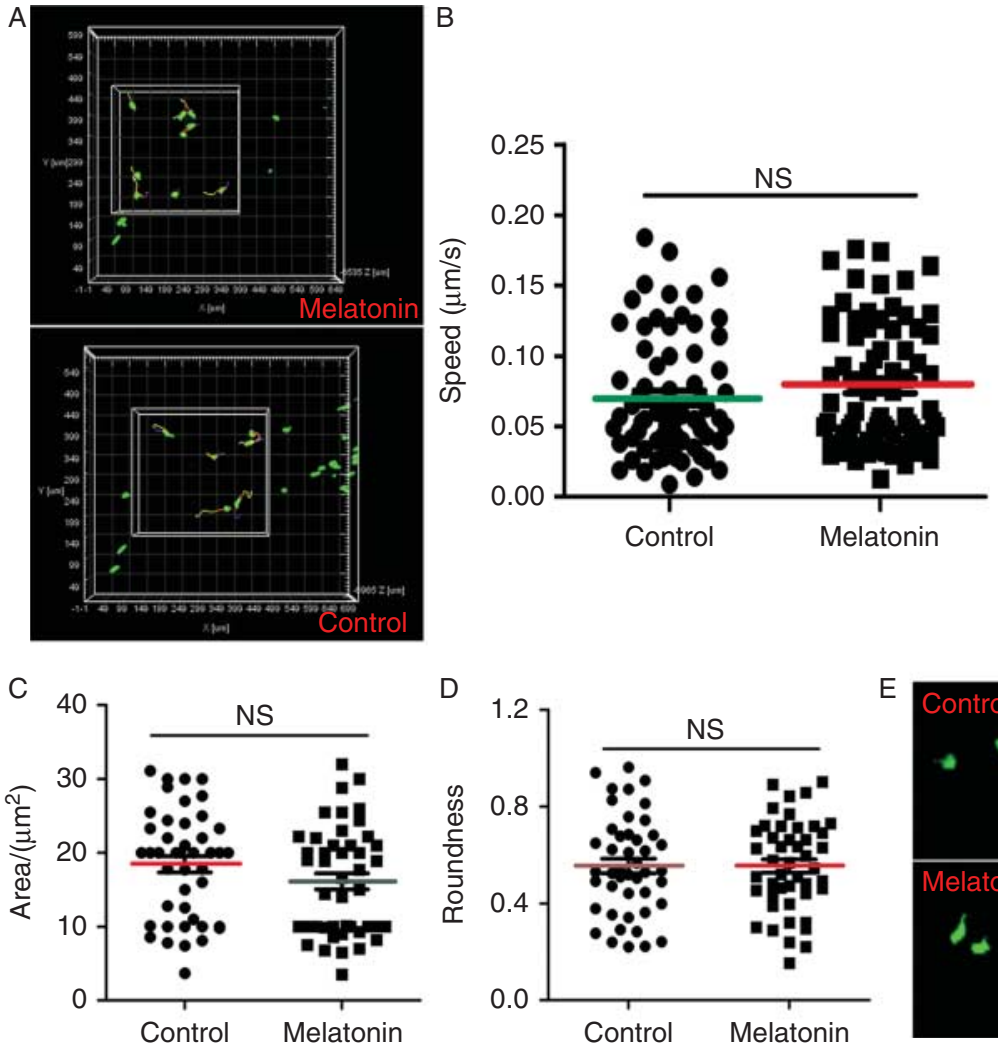

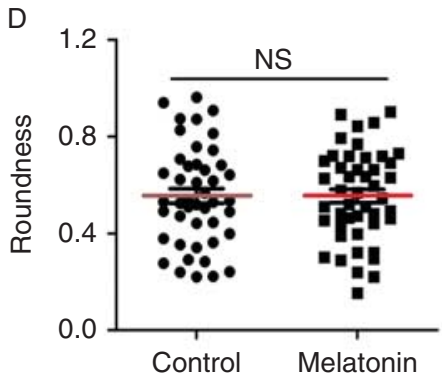

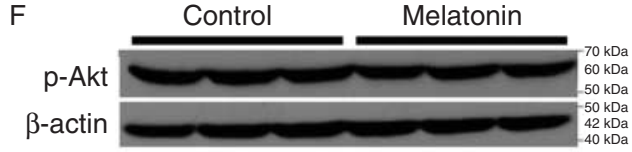

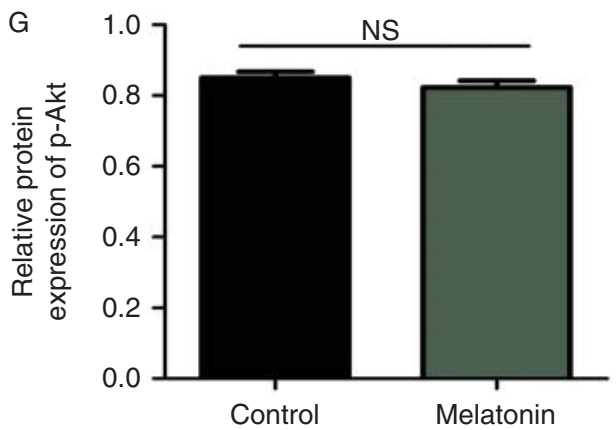

Figure 2

Melatonin did not affect the neutrophil migration activity in the tail fin tissues. ( $A$ and B) Embryo was embedded in low-melting-point agarose at $0.5 \mathrm{~h}$ post injury, and imaging was monitored for $30 \mathrm{~min}$. The analysis of motion speed on neutrophils by Imaris Software indicated no significant difference between the melatonin treatment and the control group. (C) At $1 \mathrm{~h}$ post injury, the single neutrophil area was measured by ImagJ Software. Melatonin did not induce a notable area plasticity compared with the control group. (D) The roundness of the neutrophils was also calculated as $4 *$ area/ $\pi^{*}$ major_axis ${ }^{2}$ at $1 \mathrm{~h}$ post wounding. The results indicated no significant difference in the roundness of neutrophils between the melatonin treatment and the control group. (E) Pictures showed the dynamic movement of neutrophils in $6 \mathrm{~min}$. ( $F$ and G) Western blot showed that melatonin did not affect the protein expression of p-Akt. http://joe.endocrinology-journals.org DOI: $10.1530 /$ JOE-15-0329
(C) 2015 Society for Endocrinology Printed in Great Britain
Published by Bioscientifica Ltd 
A

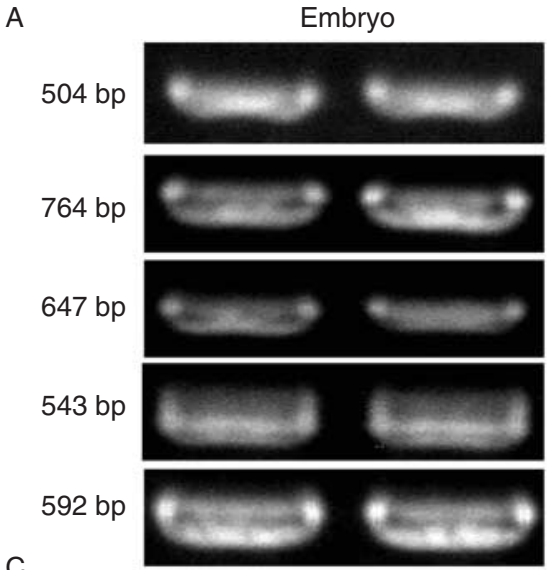

B

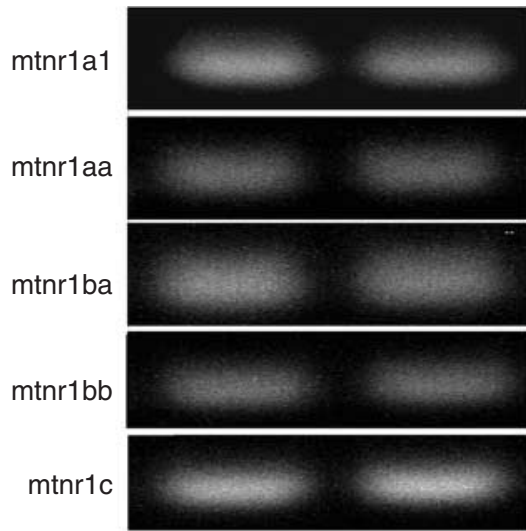

$\mathrm{D}$

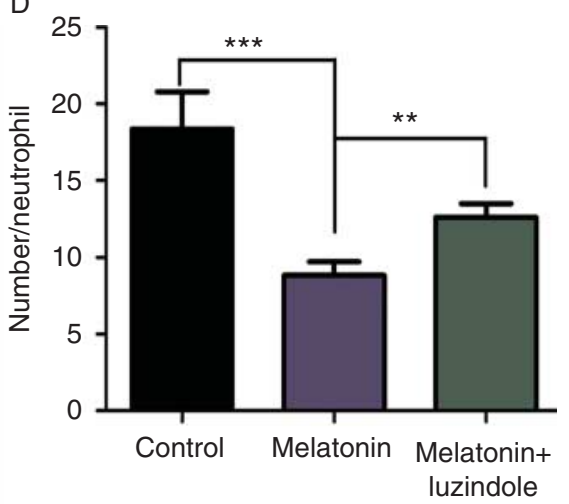

$\mathrm{E}$

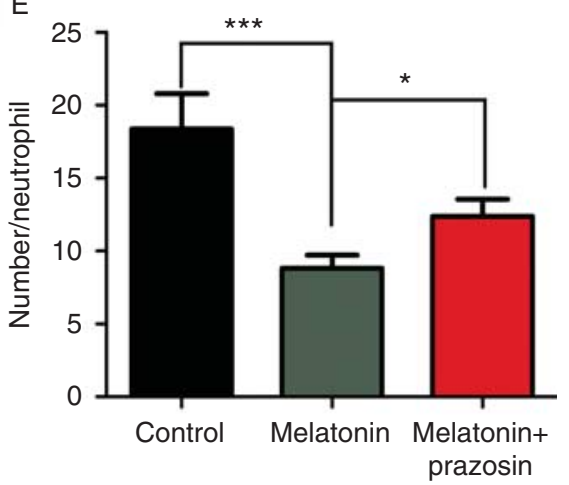

\section{Figure 3}

Melatonin receptors 1,2 , and 3 participated in the neutrophil migration process. (A) A zebrafish embryo was homogenized to identify the melatonin receptor subtypes using RT-PCR. Melatonin receptor subtypes 1a1, 1aa, 1ba, 1bb, and 1c were all detected at the whole mount level. (B) The total RNA of the blood sample collected from adult zebrafish after treatment with red blood cell lysis buffer was extracted using trizol to detect the melatonin receptor in the leukocytes. The leukocytes also

luzindole for $1 / 2$ (Rawashdeh et al. 2007) and prazosin for 3 (Requintina \& Oxenkrug 2007), to block the melatonin effect. We found that both luzindole and prazosin could suppress the promoted migration capacity of melatonin (Fig. 3C, D and E). These results demonstrated that melatonin receptors 1,2 , and 3 , especially expressed in leukocytes, all acted in the injury-induced neutrophil migration.

Given the role of the MAPK family, JNK, p38 (Taylor et al. 2013), and ERK (Yang et al. 2014), in regulating cell migration, we supposed that melatonin may regulate ERK activity in the zebrafish injury model. Western blot showed that melatonin obviously inhibited ERK protein phosphorylation, which was blocked by its receptor antagonists (Fig. 4A and B). The result also showed that green fluorescent protein-labeled neutrophils co-localized with DAPI were stained with p-ERK and that melatonin significantly impaired the p-ERK expression in neutrophils expressed the five melatonin receptor subtypes at the mRNA level. (C) Melatonin receptor antagonists luzindole (MT1/MT2) and prazosin (MT3) were used to evaluate the role of the melatonin receptor in neutrophil migration in the injury model. Both luzindole (D) and prazosin (E) could partially block the inhibited migration effect caused by melatonin ( $* P<0.05, * * P<0.01, * * * P<0.001, t$-test and ANOVA analysis).

(Fig. 4C and D). Both detection methods collectively identified that exogenous melatonin significantly suppressed the injury-induced ERK protein activity at the total and cellular levels.

Here, we speculated on whether ERK plays a role in neutrophil migration. The result showed that ERK activity at the total protein level was almost completely inhibited within $1 \mathrm{~h}$ after PD0325901, an MEK inhibitor, administration compared with that in the normal group (Fig. 5B). The single neutrophil staining collected from the treated blood of zebrafish also exhibited the similar blocked consequence of PD0325901 on ERK activity (Fig. 5A and C). Moreover, PD0325901 resulted in a dramatically faint neutrophil migration ability compared with the control (Fig. 5C), consistent with the previous report in mice (Mizuno et al. 2014). Then, to remedy the p-ERK deficiency caused by melatonin, EGF was used in combination with melatonin to recover the ERK function

Published by Bioscientifica Ltd 
A

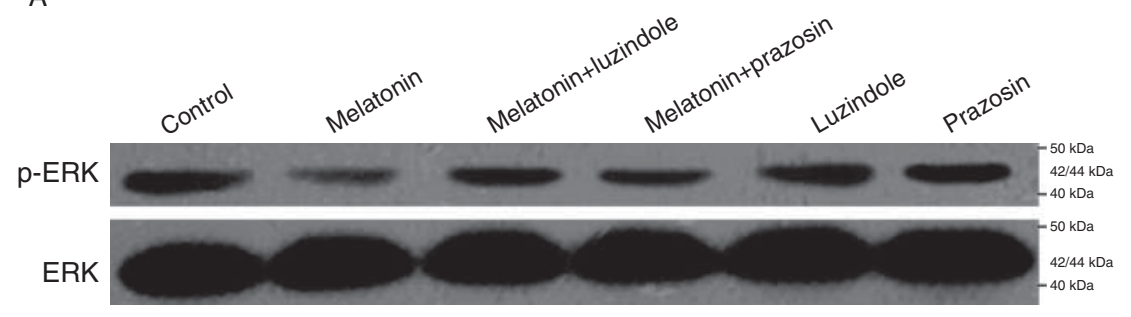

C
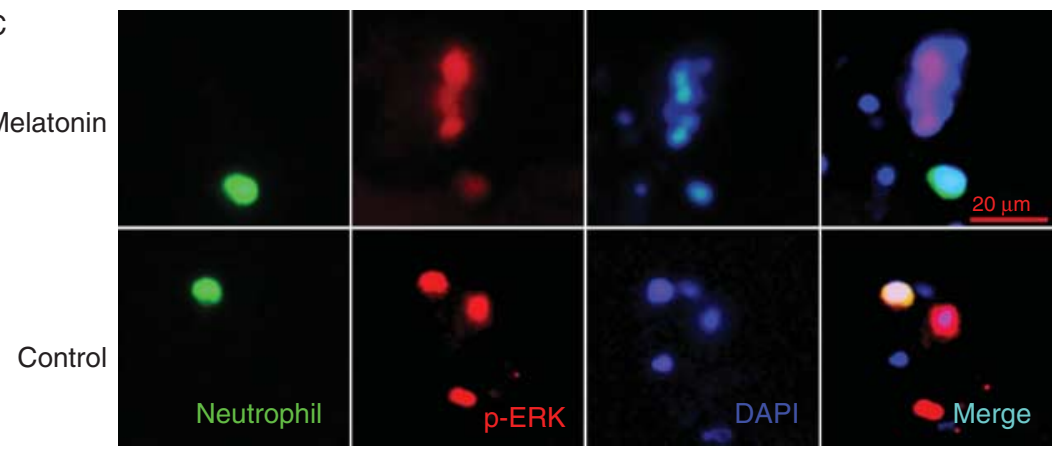
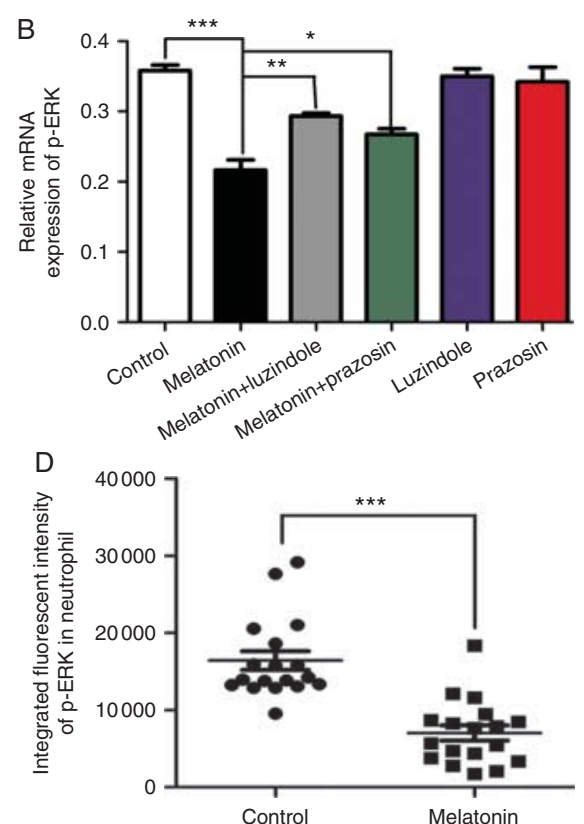

\section{Figure 4}

Melatonin suppressed the injury-induced ERK protein phosphorylation. ( $A$ and $B$ ) About $1 \mathrm{~h}$ post injury, the embryo was collected for total protein analysis using western blot. The result showed that melatonin treatment significantly suppressed the ERK protein (42/44 kd) phosphorylation level post injury, which was blocked by its receptor antagonist, luzindole and prazosin. (C and D) Blood sampled from adult transgenic Tg(lyz:EGFP)

(Grande et al. 2002, Amos et al. 2006). Western blot showed that EGF combined with melatonin treatment significantly enhanced the ERK activity compared with melatonin alone (Fig. 5D). Moreover, to some extent, EGF increased the number of migrating neutrophils to the injury site (Fig. 5E). Therefore, we can conclude that ERK, a vital phosphokinase in various processes, also has functional involvement as a way to regulate neutrophil recruitment toward the injury site.

Because ERK plays a vital role to regulate neutrophil recruitment, we next asked which step of the recruitment cascade was affected by MEK inhibitor, PD0325901. Given the transgenic zebrafish line labeled as both vessel and neutrophil, we were able to explore the process of neutrophils adhering and transmigrating to vessel endothelial cells (Fig. 6A). Zebrafish tissues near the cloacal aperture were injured by sterile needle (Fig. 6B), and the dynamic transmigrated behavior was continuously scanned as shown in Fig. 6C. When treated with PD0325901 and melatonin, a smaller number of neutrophils could crawl over to the vessel endothelial cells after injury compared with the control (Fig. 6D). Subsequently, we evaluated the effect of PD0325901 and melatonin on zebrafish underwent immunofluorescent assay. Transgenic neutrophils labeled by EGFP were co-localized with p-ERK (red) and DAPI (blue). Moreover, a weaker fluorescence intensity of $p$-ERK in the neutrophils appeared with melatonin treatment unlike in the control group ( ${ }^{*} P<0.05$, $\star * P<0.01, * * * P<0.001, t$-test and ANOVA analysis).

the total number of circulating neutrophils. The result indicated that hardly any difference in the total number of neutrophils was found between the PD0325901, melatonin, and the control groups (Fig. 6E). Next, we examined whether the migrated-related adhesion molecule was influenced by PD0325901 and melatonin. The results indicated that PD0325901 significantly inhibited the expression of both P-selectin and E-selectin and that melatonin could also suppress the expression of E-selectin (Fig. 6F and G). Overall, the results demonstrated that the inhibition of ERK resulted in a more difficult process of crawling over to the vessel endothelial cells of neutrophils. This process could be meditated by regulating the related adhesion molecular expression.

\section{Discussion}

In zebrafish, melatonin is synthesized mainly from the pineal gland and the retina, and it is evolutionarily and highly conserved in sequence and function among many vertebrates. It plays a central circadian-controlling role in various physiological activities, including sleep keeping, memory formation, locomotor activity, and

Published by Bioscientifica Ltd. 
A

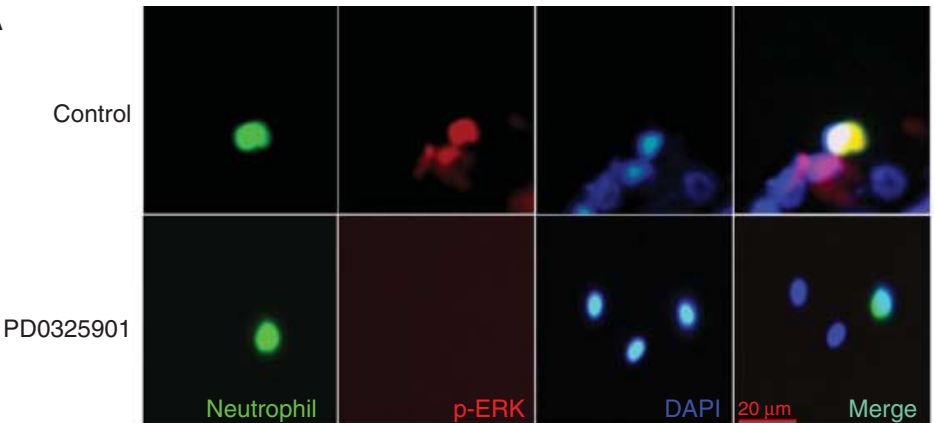

C

D
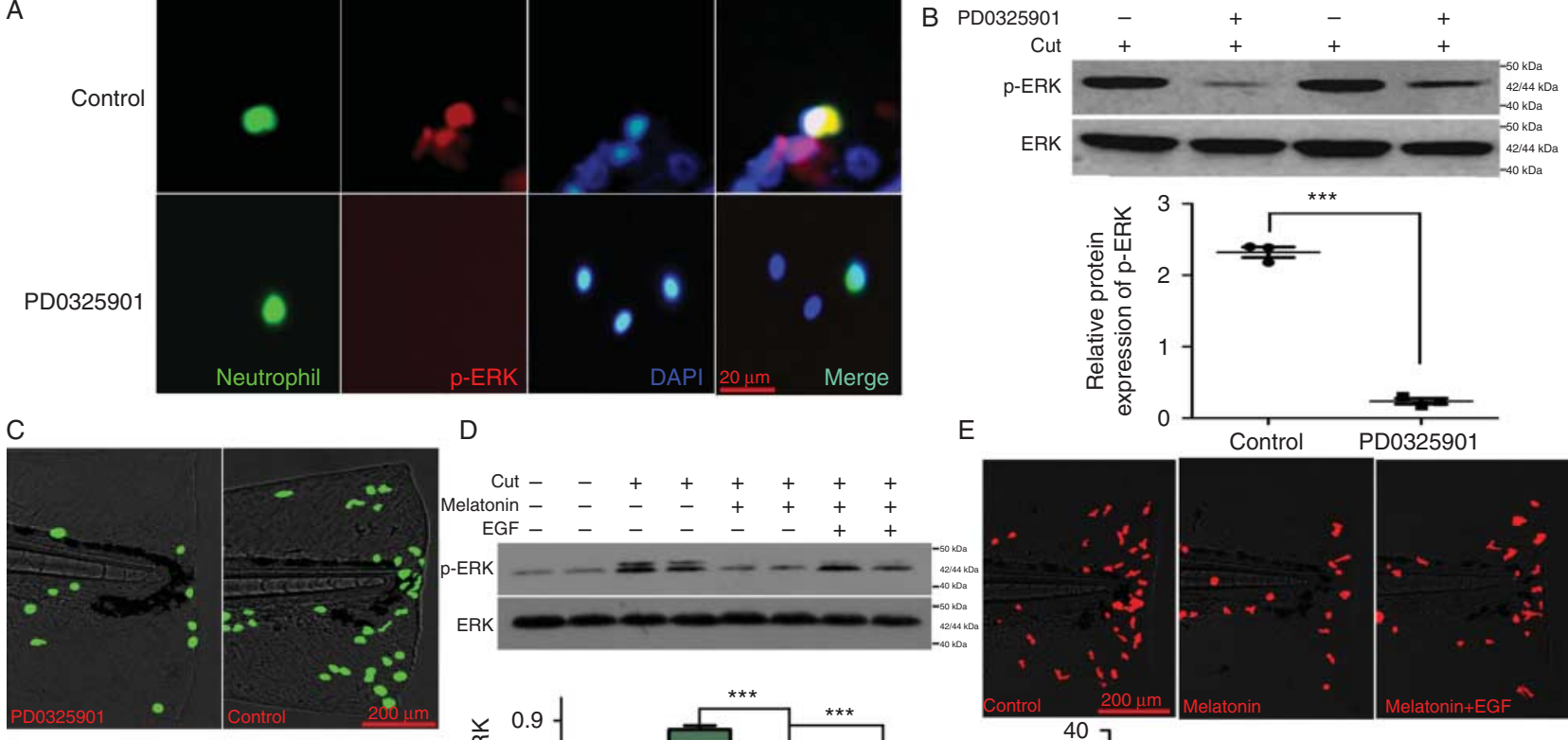

E
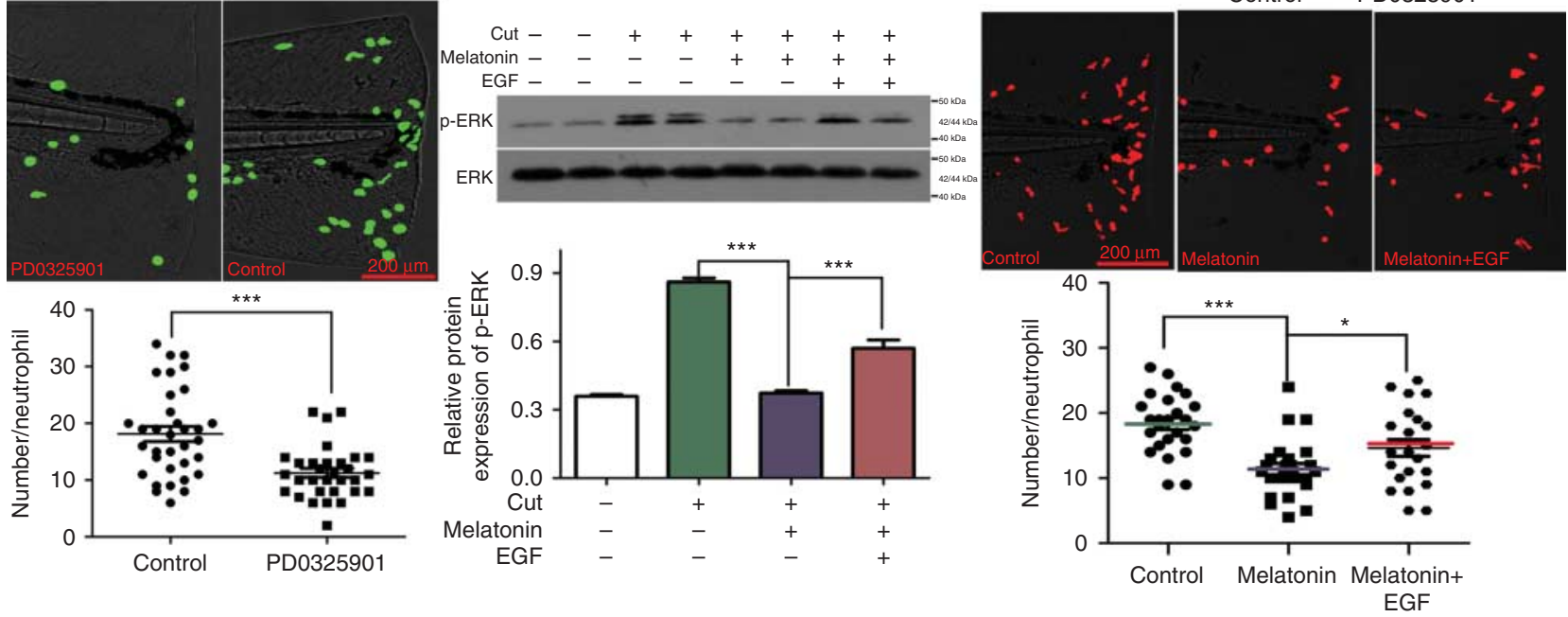

Figure 5

ERK regulated neutrophil recruitment toward the injury site. PD0325901 and EGF were brought into the experiment to block and enhance ERK activity similar to a previous study. (A and B) PD0325901 treatment during injury almost completely inhibited the ERK phosphorylation at the total protein level (B). At the immunofluorescent level, PD0325901 also significantly suppressed the p-ERK expression in a single neutrophil (A). (C) Using the same concentration treatment, PD0325901 inhibited

reproductive behavior, which coordinate organisms to adapt to the changes in the surrounding environment (Lima-Cabello et al. 2014).

In the current study, we evaluated in vivo the antiinflammatory effect of melatonin on neutrophil migration to the injury-induced stimulus. Neutrophils were rapidly recruited to the injury site post injury, peaked at $\sim 6 \mathrm{~h}$, and dismissed at $\sim 24 \mathrm{~h}$ (Fig. $1 \mathrm{~A}, \mathrm{~B}$ and $\mathrm{C}$ ). Using the model, we examined the role of exogenous melatonin in neutrophil migration at $3 \mathrm{~h}$ post injury. The results showed that exogenous melatonin obviously inhibited the neutrophil migration in a dose- and time-dependent manner (Fig. 1E and G). Although the administrated concentration of melatonin was higher than the physiological level in zebrafish, the actually effective concentration would decrease dramatically using micro-injection

neutrophil recruitment toward the injury area. (D) Embryo was treated with EGF and melatonin together and with melatonin alone. EGF could remedy the deficiency of ERK phosphorylation caused by melatonin.

(E) EGF together with melatonin also induced increased neutrophil migration compared with melatonin treatment alone $(* P<0.05$, $* * * P<0.001, t$-test and ANOVA analysis).

treatment instead of the water-delivery way. Taking advantage of the model, we further detected the dynamic movement behavior of neutrophils to analyze if their activity was regulated by melatonin. During the course of $0.5 \mathrm{~h}$ of live imaging, the neutrophils were monitored to migrate to the tail fin tissues. The analysis indicated that the motion speed of neutrophils had no significant difference between the melatonin treatment and the control group. This motion activity was also indexed by the area and roundness of neutrophils. Overall, melatonin only regulated the number of neutrophils that were recruitment but did not affect their motion activity, such as speed and roundness, in the caudal fin. Here, we supposed that melatonin just impaired the directional neutrophils migration toward the injury, but did not affect the random cell motility. Previous studies demonstrated 


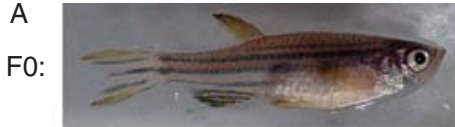

$\operatorname{Tg}(\operatorname{lyz}: D s R e d)$

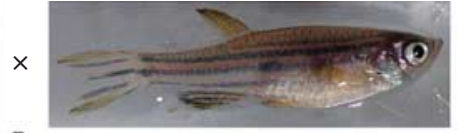

$\operatorname{Tg}(f l k: E G F P)$

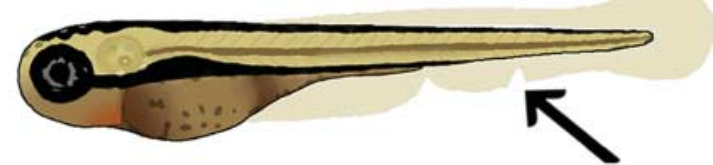

F1:

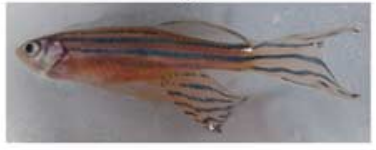

$\operatorname{Tg}($ lyz:DsRed);(flk:EGFP)

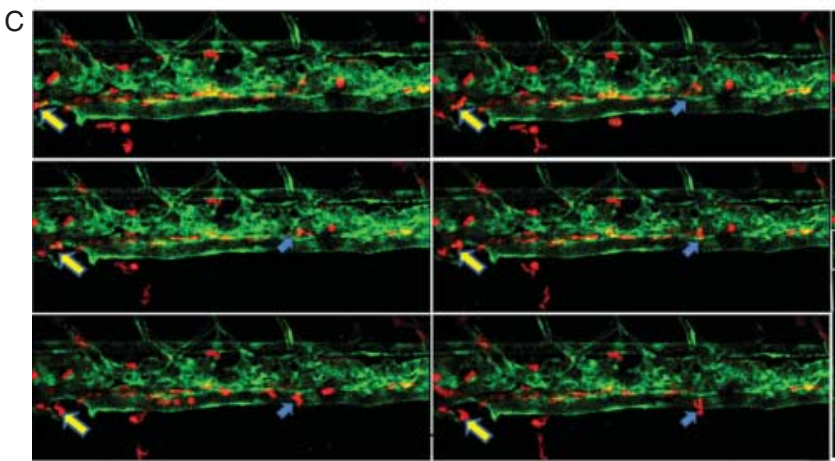

D
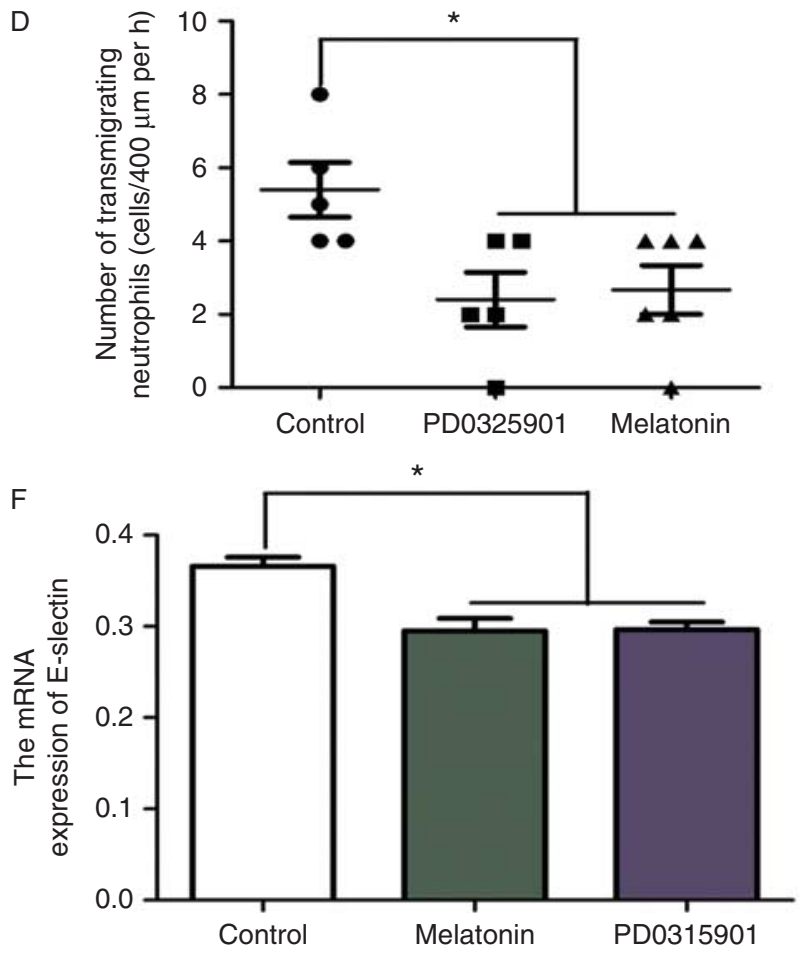

Figure 6

ERK regulated neutrophils transmigrating to endothelial cells. (A) Double transgenic zebrafish labeled as both vessel and neutrophil was harvested through the hybridization of $\mathrm{Tg}(f l k: \mathrm{EGFP})$ and $\mathrm{Tg}(\mathrm{lyz}$ :DsRed) lines.

(B) Embryo tissue near the cloacal orifice was injured by a sterile blade to create an acute inflammation model. (C) The dynamic behavior of neutrophils crawling and transmigrating to the vessel endothelial cells was monitored for $0.5 \mathrm{~h}$. The arrow shows the representative neutrophil that successfully crossed over to the vessel endothelial cells. (D) PD0325901

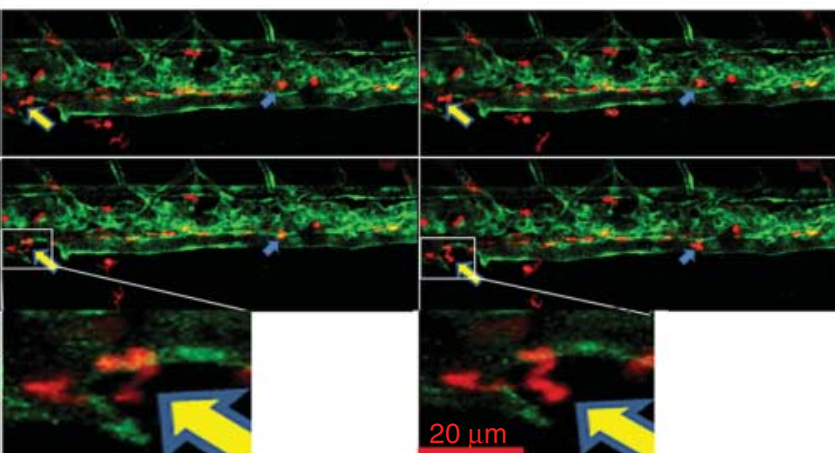

$E$
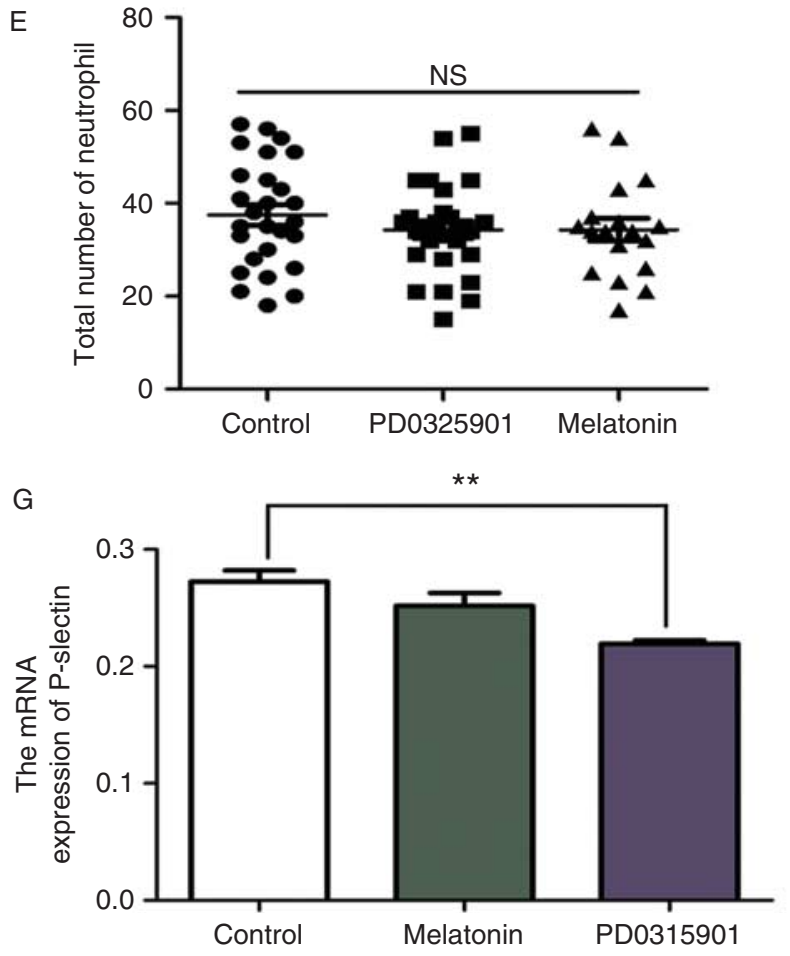

significantly inhibited the neutrophils transmigrating to the vessel. (E) PD0325901 did not cause the change in the total number of circulating neutrophils. The neutrophils located in the $800 \mu \mathrm{m}$ range from the spinal cord terminal to the head were counted as the total number. ( $F$ and $G$ ) PD0325901 and melatonin inhibited the related adhesion molecules, P-selectin and E-selectin, expressed at the mRNA level $(* P<0.05, * * P<0.01$, $t$-test and ANOVA analysis).

Published by Bioscientifica Ltd. 
that melatonin could play a positive (Pena et al. 2007, Ren et al. 2015) or negative (Lotufo et al. 2001) effect in neutrophil function. By incorporating the inconsistent effects of melatonin in previous studies (Lotufo et al. 2001, Pena et al. 2007), we inferred that the ultimate role of melatonin could result from the initial pathophysiological conditions and the various application concentrations.

Subsequently, we wanted to determine if a melatonin receptor participated in the process, and if so, which one. Using a specific primer, we found that melatonin receptors $1 \mathrm{a}, 1 \mathrm{~b}$, and $1 \mathrm{c}$ were all expressed in the embryo and especially in the leukocytes (Fig. 3A and B). Then, the melatonin receptor antagonists luzindole (MT1/MT2) and prazosin (MT3) were adopted to analyze the receptor function. Both receptor antagonists could block the inhibited migration effect of melatonin on neutrophils (Fig. 3C and D). Indeed, the melatonin receptor was involved in the migration process with melatonin treatment. Moreover, we aimed to reveal the potential mechanism of melatonin in neutrophil migration. MAPK/ERK proteins regulate many inflammatory events related to neutrophils, such as extracellular trap formation and adhesion to the endothelial cell monolayer (Pillinger et al. 1998, Rosenthal et al. 1998, Hakkim et al. 2011). Thus, we detected the influence of melatonin on the p-ERK protein expression level in zebrafish. The results showed that melatonin dramatically suppressed the ERK phosphorylation with melatonin treatment at the total protein level and the cellular level post tail fin injury (Fig. 4A and C). Whether ERK protein played a role in neutrophil migration became the main issue. We found that PD0325901, an MEK inhibitor, could significantly block the ERK phosphorylation using western blot and immunohistochemistry detection (Fig. 5A and C). With PD0325901 treatment, neutrophils exhibited faint migration ability to the injury site, as reflected in the migrating number (Fig. 5C and D). Finally, the cascaded steps of neutrophil migration were spectated using transgenic zebrafish labeled as both vessel and neutrophil to evaluate the ERK effect on neutrophil behavior. In vivo imaging revealed that PD0325901 significantly inhibited neutrophils transmigrating across the vessel endothelial cells (Fig. 6C and D). PD0325901 did not decrease the total number of neutrophils in the vessel (Fig. 6E). We also found that both PD0325901 and melatonin could inhibit migrated-related adhesion molecular expression. Therefore, we conclude that ERK may affect the expression of neutrophils or endothelial cell adhesion molecules. Thus, the adherence of neutrophils to the endothelial cells may loosen and result in the difficult transmigration across the vessel of neutrophils.

Neutrophil migration responding to outside stimulus is an immediate event of innate immunity among organisms against pathogen invasion. Although an appropriate recruitment level of immune cells is beneficial for human homeostasis, excessive and prolonged inflammation such as chronic inflammation could lead to repeated tissue damage and a brutal tissue environment. Melatonin, as a powerful antioxidant (Takayama et al. 1998) that scavenges different types of free radicals in the body, has been extensively administrated to regulate sleeping and aging. Our study demonstrated in vivo that exogenous melatonin could significantly suppress the inflammatory response, especially in neutrophil migration to the injury site, in a concentrated and time-dependent manner. Thus, a significant correlation was found between the endocrine hormone and immune events. According to a previous study, melatonin may be administrated to regulate an inflammation event and human health as an exogenous, non-toxic, and easily synthesized pharmacological reagent to avoid some side effects of other chemical agents (Radogna et al. 2010).

\section{Supplementary data}

This is linked to the online version of the paper at http://dx.doi.org/10.1530/ JOE-JOE-15-0329.

\section{Declaration of interest}

The authors declare that there is no conflict of interest that could be perceived as prejudicing the impartiality of the research reported.

\section{Funding}

This research was supported by a grant from the National Basic Research Program of China (973) (2012CB947602).

\section{Acknowledgements}

We thank our Core Facility Center for Life Sciences, University of Science and Technology of China.

\section{References}

Amos S, Redpath GT, Polar G, McPheson R, Schiff D \& Hussaini IM 2006 Farnesylthiosalicylic acid induces caspase activation and apoptosis in glioblastoma cells. Cell Death and Differentiation 13 642-651. (doi:10.1038/sj.cdd.4401783)

Appelbaum L, Vallone D, Anzulovich A, Ziv L, Tom M, Foulkes NS \& Gothilf Y 2006 Zebrafish arylalkylamine-N-acetyltransferase genestargets for regulation of the circadian clock. Journal of Molecular Endocrinology 36 337-347. (doi:10.1677/jme.1.01893)

Published by Bioscientifica Ltd. 
Barrett P \& Bolborea M 2012 Molecular pathways involved in seasonal body weight and reproductive responses governed by melatonin. Journal of Pineal Research 52 376-388. (doi:10.1111/j.1600-079X.2011. 00963.x)

Baydas G, Ozveren F, Akdemir I, Tuzcu M \& Yasar A 2005 Learning and memory deficits in rats induced by chronic thinner exposure are reversed by melatonin. Journal of Pineal Research 39 50-56. (doi:10.1111/j.1600-079X.2005.00212.x)

Blalock JE 1994 The syntax of immune-neuroendocrine communication. Immunology Today 15 504-511. (doi:10.1016/0167-5699(94)90205-4)

Choi J, Dong L, Ahn J, Dao D, Hammerschmidt M \& Chen JN 2007 FoxH1 negatively modulates flk1 gene expression and vascular formation in zebrafish. Developmental Biology 304 735-744. (doi:10.1016/j.ydbio. 2007.01.023)

Danilova N, Krupnik VE, Sugden D \& Zhdanova IV 2004 Melatonin stimulates cell proliferation in zebrafish embryo and accelerates its development. FASEB Journal 18 751-753. (doi:10.1096/fj.03-0544fje)

De Borsetti NH, Dean BJ, Bain EJ, Clanton JA, Taylor RW \& Gamse JT 2011 Light and melatonin schedule neuronal differentiation in the habenular nuclei. Developmental Biology 358 251-261. (doi:10.1016/j.ydbio. 2011.07.038)

Delgobbo V, Libri V, Villani N, Calio R \& Nistico G 1989 Pinealectomy inhibits interleukin-2 production and natural-killer activity in mice. International Journal of Immunopharmacology 11 567-573. (doi:10.1016/ 0192-0561(89)90187-2)

Elbaz I, Foulkes NS, Gothilf Y \& Appelbaum L 2013 Circadian clocks, rhythmic synaptic plasticity and the sleep-wake cycle in zebrafish. Frontiers in Neural Circuits 7 183-189. (doi:10.3389/fncir.2013.00009)

Falcon J, Galarneau KM, Weller JL, Ron B, Chen G, Coon SL \& Klein DC 2001 Regulation of arylalkylamine $\mathrm{N}$-acetyltransferase-2 (AANAT2, EC 2.3.1.87) in the fish pineal organ: evidence for a role of proteasomal proteolysis. Endocrinology 142 1804-1813. (doi:10.1210/ endo.142.5.8129)

Fusani L \& Gahr M 2014 Differential expression of melatonin receptor subtypes MelIa. MelIb and MelIc in relation to melatonin binding in the male songbird brain. Brain, Behavior and Evolution 85 4-14. (doi:10.1159/000367984)

Germena G \& Hirsch E 2013 PI3Ks and small GTPases in neutrophil migration: two sides of the same coin. Molecular Immunology 55 83-86. (doi:10.1016/j.molimm.2012.10.004)

Grande M, Franzen A, Karlsson JO, Ericson LE, Heldin NE \& Nilsson M 2002 Transforming growth factor- $\beta$ and epidermal growth factor synergistically stimulate epithelial to mesenchymal transition (EMT) through a MEK-dependent mechanism in primary cultured pig thyrocytes. Journal of Cell Science 115 4227-4236. (doi:10.1242/jcs.00091)

Hakkim A, Fuchs TA, Martinez NE, Hess S, Prinz H, Zychlinsky A \& Waldmann H 2011 Activation of the Raf-MEK-ERK pathway is required for neutrophil extracellular trap formation. Nature Chemical Biology 7 75-77. (doi:10.1038/nchembio.496)

Haldar C, Haussler D \& Gupta D 1992 Effect of the pineal-gland on circadian rhythmicity of colony-forming-units for granulocytes and macrophages (Cfu-Gm) from rat bone-marrow cell-cultures. Journal of Pineal Research 12 79-83. (doi:10.1111/j.1600-079X.1992.tb00030.x)

Kazimi N \& Cahill GM 1999 Development of a circadian melatonin rhythm in embryonic zebrafish. Brain Research. Developmental Brain Research 117 47-52. (doi:10.1016/S0165-3806(99)00096-6)

Kleber A, Altmeyer S, Wolf B, Wolf A, Volk T, Fink T \& Kubulus D 2014 Impact of melatonin receptor deletion on intracellular signaling in spleen cells of mice after polymicrobial sepsis. Inflammation Research 63 1023-1033. (doi:10.1007/s00011-014-0779-4)

Lee SJ, Jung YH, Oh SY, Yun SP \& Han HJ 2014 Melatonin enhances the human mesenchymal stem cells motility via melatonin receptor 2 coupling with $\mathrm{G} \alpha \mathrm{q}$ in skin wound healing. Journal of Pineal Research $\mathbf{5 7}$ 393-407. (doi:10.1111/jpi.12179)

Li L, Yan B, Shi YQ, Zhang WQ \& Wen ZL 2012 Live imaging reveals differing roles of macrophages and neutrophils during zebrafish tail fin regeneration. Journal of Biological Chemistry 287 25353-25360. (doi:10.1074/jbc.M112.349126)

Lima-Cabello E, Diaz-Casado ME, Guerrero JA, Otalora BB, Escames G, Lopez LC, Reiter RJ \& Acuna-Castroviejo D 2014 A review of the melatonin functions in zebrafish physiology. Journal of Pineal Research 57 1-9. (doi:10.1111/jpi.12149)

Liu F \& Wen ZL 2002 Cloning and expression pattern of the lysozyme C gene in zebrafish. Mechanisms of Development 113 69-72. (doi:10.1016/ S0925-4773(01)00658-X)

Lotufo CMC, Lopes C, Dubocovich ML, Farsky SHP \& Markus RP 2001 Melatonin and $\mathrm{N}$-acetylserotonin inhibit leukocyte rolling and adhesion to rat microcirculation. European Journal of Pharmacology 430 351-357. (doi:10.1016/S0014-2999(01)01369-3)

Martins E, de Oliveira APL, de Araujo AMF, de Lima WT, Cipolla-Neto J \& Rosa LFBPC 2001 Melatonin modulates allergic lung inflammation. Journal of Pineal Research 31 363-369. (doi:10.1034/j.1600-079X.2001. 310412.x)

Masana MI \& Dubocovich ML 2001 Melatonin receptor signaling: finding the path through the dark. Science's STKE : Signal Transduction knowledge Environment $\mathbf{2 0 0 1}$ pe39.

Matsumoto Y, Mishima K, Satoh K, Tozawa T, Mishima Y, Shimizu T \& Hishikawa Y 2001 Total sleep deprivation induces an acute and transient increase in NK cell activity in healthy young volunteers. Sleep 24 804-809.

Mizuno R, Kamioka Y, Kabashima K, Imajo M, Sumiyama K, Nakasho E, Ito T, Hamazaki Y, Okuchi Y, Sakai Y et al. 2014 In vivo imaging reveals PKA regulation of ERK activity during neutrophil recruitment to inflamed intestines. Journal of Experimental Medicine 211 1123-1136. (doi:10.1084/jem.20132112)

Moriya T, Horie N, Mitome M \& Shinohara K 2007 Melatonin influences the proliferative and differentiative activity of neural stem cells. Journal of Pineal Research 42 411-418. (doi:10.1111/j.1600-079X.2007. 00435.x)

Pena C, Rincon J, Pedreanez A, Viera N \& Mosquera J 2007 Chemotactic effect of melatonin on leukocytes. Journal of Pineal Research 43 263-269. (doi:10.1111/j.1600-079X.2007.00471.x)

Pillinger MH, Capodici C, Rosenthal P, Kheterpal N, Hanft S, Philips MR \& Weissmann G 1998 Modes of action of aspirin-like drugs: salicylates inhibit Erk activation and integrin-dependent neutrophil adhesion. PNAS 95 14540-14545. (doi:10.1073/pnas.95. 24.14540)

Radogna F, Diederich M \& Ghibelli L 2010 Melatonin: a pleiotropic molecule regulating inflammation. Biochemical Pharmacology $\mathbf{8 0}$ 1844-1852. (doi:10.1016/j.bcp.2010.07.041)

Rawashdeh O, de Borsetti NH, Roman G \& Cahill GM 2007 Melatonin suppresses nighttime memory formation in zebrafish. Science $\mathbf{3 1 8}$ 1144-1146. (doi:10.1126/science.1148564)

Reiter RJ, Blask DE, Johnson LY, Rudeen PK, Vaughan MK \& Waring PJ 1976 Melatonin inhibition of reproduction in male hamster- its dependency on time of day of administration and on an intact and sympathetically innervated pineal-gland. Neuroendocrinology 22 107-116. (doi:10.1159/ 000122616)

Ren DL, Li YJ, Hu BB, Wang H \& Hu B 2015 Melatonin regulates the rhythmic migration of neutrophils in live zebrafish. Journal of Pineal Research 58 452-460. (doi:10.1111/jpi.12230)

Reppert SM, Weaver DR, Cassone VM, Godson C \& Kolakowski LF 1995 Melatonin receptors are for the birds - molecular analysis of 2 receptor subtypes differentially expressed in chick brain. Neuron 15 1003-1015. (doi:10.1016/0896-6273(95)90090-X)

Requintina PJ \& Oxenkrug GF 2007 Effect of luzindole and other melatonin receptor antagonists on iron- and lipopolysaccharide-induced lipid peroxidation in vitro. Neuroprotective Agents: Eighth International Neuroprotection Society Meeting 1122 289-294.

Rosenthal P, Capodici C, Weissmann G \& Pillinger MH 1998 Mechanisms of neutrophil adhesion: Inhibitors of ERK block neutrophil aggregation 
and adhesion to endothelial cell monolayers. Arthritis and Rheumatism 41 S243-S243.

Scheff JD, Calvano SE, Lowry SF \& Androulakis IP 2010 Modeling the influence of circadian rhythms on the acute inflammatory response. Journal of Theoretical Biology 264 1068-1076. (doi:10.1016/j.jtbi.2010. 03.026)

Takayama H, Caccitoio JA, Hisamochi K, Pearson PJ \& Schaff HV 1998 EPC: a novel free-radical scavenger, prevents hydroxyl radical-induced coronary artery endothelial damage and stimulates endogenous intimal nitric oxide release. Circulation 98 176-176.

Taylor HB, Liepe J, Barthen C, Bugeon L, Huvet M, Kirk PD, Brown SB, Lamb JR, Stumpf MP \& Dallman MJ 2013 P38 and JNK have opposing effects on persistence of in vivo leukocyte migration in zebrafish. Immunology and Cell Biology 91 60-69. (doi:10.1038/icb.2012.57)
Tell RM, Kimura K \& Palic D 2012 Rac2 expression and its role in neutrophil functions of zebrafish (Danio rerio). Fish \& Shellfish Immunology 33 1086-1094. (doi:10.1016/j.fsi.2012.07.020)

Yang L, Zheng JC, Xu R, Zhang YJ, Gu L, Dong J, Zhu YC, Zhou RJ, Zheng L, Zhang XY et al. 2014 Melatonin suppresses hypoxia-induced migration of HUVECs via inhibition of ERK/Rac1 activation. International Journal of Molecular Sciences 15 14102-14121. (doi:10.3390/ ijms150814102)

Yoo SK, Lam PY, Eichelberg MR, Zasadil L, Bement WM \& Huttenlocher A 2012 The role of microtubules in neutrophil polarity and migration in live zebrafish. Journal of Cell Science 125 5702-5710. (doi:10.1242/jcs. 108324)

Zhdanova IV 2011 Sleep and its regulation in zebrafish. Reviews in the Neurosciences 22 27-36. (doi:10.1515/rns.2011.005)

Received in final form 21 August 2015

Accepted 24 August 2015

Accepted Preprint published online 24 August 2015
Published by Bioscientifica Ltd. 